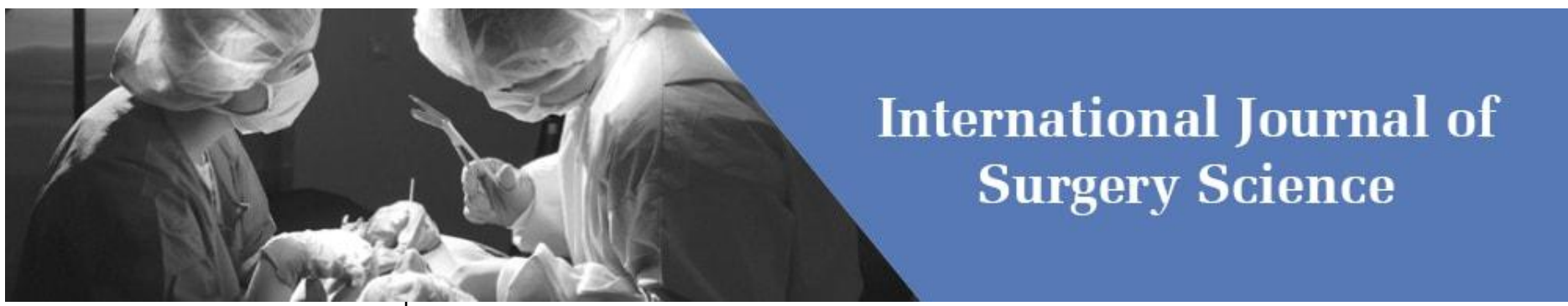

E-ISSN: 2616-3470

P-ISSN: 2616-3462

(C) Surgery Science

www.surgeryscience.com

$2020 ; 4(3): 205-207$

Received: 02-05-2020

Accepted: 04-06-2020

Dr. Sreekaraswamy R Sumukha Assistant Professor, Department of surgery, Siddhartha Institute of

Medical Sciences Hospital \& Research Center, T Begur,

Karnataka, India
Corresponding Author: Dr. Sreekaraswamy R Sumukha Assistant Professor, Department of surgery, Siddhartha Institute of

Medical Sciences Hospital \&

Research Center, T Begur,

Karnataka, India

\section{The advantage of near-total thyroidectomy to avoid postoperative hypoparathyroidism in benign multinodular goiter}

\section{Dr. Sreekaraswamy R Sumukha}

DOI: https://doi.org/10.33545/surgery.2020.v4.i3d.491

\section{Abstract}

Introduction: In recent years, total or near-total thyroidectomy has emerged as a surgical option to treat patients with multinodular goiter, especially in endemic iodine-deficient regions. The aim of this study was to compare the complication rates of total and near-total thyroidectomy in multinodular goiter and the incidence of thyroid cancer requiring radioactive iodine ablation and completion thyroidectomy between groups.

Study Design: Patients with euthyroid multinodular goiter without any preoperative suspicion of malignancy, history of familial thyroid cancer, or previous exposure to radiation were randomized (according to a random table) to total thyroidectomy group 1, and near-total thyroidectomy leaving less than $2 \mathrm{gm}$. Study period was between November 2018 to October 2019 at SSIMS, T. BEGUR.

Keywords: thyroidectomy, hypoparathyroidism, multinodular goiter

\section{Introduction}

In recent years, total or near-total thyroidectomy has emerged as a surgical option to treat patients with multinodular goiter, especially in endemic iodine-deficient regions. The aim of this study was to compare the complication rates of total and near-total thyroidectomy in multinodular goiter and the incidence of thyroid cancer requiring radioactive iodine ablation and completion thyroidectomy between groups.

\section{Results}

Near-total thyroidectomy was the principal surgical procedure performed for benign thyroid disease. The temporary recurrent laryngeal nerve palsy rate with respect to the nerves at risk was $3.3 \%$ ( 2 of 60 nerves), whereas temporary hypoparathyroidism was $7 \%$ ( 2 of 30 patients). Neither permanent recurrent laryngeal nerve palsy nor permanent hypoparathyroidism occurred. In 1 patient, wound hematoma developed and required re-exploration. Seroma in another patient needed no medical or surgical intervention. Neither wound infection nor mortality were noted.

\section{Conclusion}

In conclusion, we recommend near-total thyroidectomy in multinodular goiter instead of total or subtotal thyroidectomy.

\section{Methods}

\section{Inclusion Criteria}

- A benign bilateral thyroid disease with the posterior aspects of both thyroid lobes appearing normal on ultrasound of the neck.

\section{Exclusion Criteria}

- thyroid disease involving the posterior aspect/s of thyroid lobe/s,

- $\quad$ suspicion of thyroid cancer,

- previous thyroid surgery,

- pregnancy or lactation,

- Age $<24$ years or $>78$ years, 
- ASA 4 grade (American Society of Anesthesiology),

- and inability to comply with the follow-up protocol.

Between Nov 2018 and October 2019, 30 patients with goiter who underwent surgical treatment in our hospital at SSIMS, T. BEGUR, were included in this study. All patients provided informed consent. All patients were evaluated by means of physical examination, thyroid function tests, thyroid ultrasonography, and thyroid scintigraphy. Fine-needle aspiration biopsy was performed for hypoactive and dominant thyroid nodules. In our surgical department, we performed total thyroidectomy in single nodules that were suspected of malignancy at fine-needle aspiration biopsy; therefore, these patients were specifically excluded. Antithyroid medications were used for hyperthyroidism before surgery to attain euthyroidism.

Video laryngostroboscopic examinations were performed to evaluate vocal cord motility 24 hours before surgery and 48 hours after surgery in all patients and included confirmation of normal vocal cord movement in patients who had temporary neuropraxia. Near-total thyroidectomy was performed in all cases and consisted of total lobectomy in the lobe having the dominant nodule, with isthmectomy and near-total lobectomy in the contralateral side, leaving a small quantity of about $2 \mathrm{gm}$ of thyroid tissue adjacent to the parathyroid glands and their blood supply. Recurrent laryngeal nerves in all cases were identified and traced to the cricoid cartilage in either side. Every attempt was made to demonstrate and preserve all of the parathyroid glands with their blood supply. When a parathyroid gland was damaged, it was transplanted into the sternocleidomastoid muscle.

In each patient, serum calcium and phosphorus levels were checked on the first and second postoperative days. Hypocalcemia is defined as low ionized serum calcium levels and the presence of symptoms of hypocalcemia. We considered hypocalcemia temporary when it lasted about 3 to 6 weeks after surgery. Calcium levels were followed up in patients who were temporarily hypocalcemic and who needed calcium supplements.

\section{Results}

There were 21 female and 9 male patients, with a mean age of 43 years (range, 24-78 years). We performed near-total thyroidectomy in all patients; the various indications are summarized in

Table 1: Indications for Surgical Treatment

\begin{tabular}{|c|c|}
\hline Indication & No. (\%) of Patients \\
\hline Suspicious or indefinite cytologic findings & 0 \\
\hline Compressive symptoms & 11 \\
\hline Recent enlargement & 3 \\
\hline Cosmesis & 3 \\
\hline Toxic multinodular goiter & 13 \\
\hline Total & 30 \\
\hline
\end{tabular}

The clinical signs are, 17 patients were treated medically because of hyperthyroidism to achieve euthyroidism preoperatively. Thyroid scintigraphy depicted hypoactive nodules in 19 patients. Fine-needle aspiration biopsy was performed in 20 patients, and the results were neither suspected nor malignant histopathologically. Intraoperative frozen section evaluations were used for nodules suspected of being malignant that had not been suspected of being malignant preoperatively; there was no malignancy at frozen section evaluation.
Parathyroid auto transplantation was performed in 2 patients, and none developed temporary or permenant hypoparathyroidism.

\section{Postoperative complications are summarized below.}

The temporary recurrent laryngeal nerve palsy rate with respect to the nerves at risk was $3.3 \%$ ( 2 of 60 nerves), whereas temporary hypoparathyroidism was $7 \%$ (2 of 30 patients). Neither permanent recurrent laryngeal nerve palsy nor permanent hypoparathyroidism occurred. Wound hematoma developed in 1 patient and required re-exploration. Seroma in another patient needed no medical or surgical intervention. 2 papillary carcinomas in 1 female patients and 1 male patient were diagnosed by using paraffin blocks. We performed iodine I 131 ablation in these patients. Neither wound infection nor mortality was noted.

\section{Discussion}

The surgical treatment of benign thyroid disease is still controversial. Many treatments have been described for the surgical management of multinodular goiter, including unilateral or bilateral subtotal thyroidectomy, hemithyroidectomy, neartotal thyroidectomy, and total thyroidectomy. Despite numerous studies of operative strategies, we still do not have clear evidence about which option is the best. When the disorder affects both lobes, total or near-total thyroidectomy is mandatory, especially in younger persons, to obviate suppressive therapy and possible relapse. Subtotal thyroidectomy may be the best elective procedure in older patients to avoid total and permanent dependence on drugs. Some authors favor the subtotal procedure in the treatment of benign multinodular disease because of its lower incidence of iatrogenic injuries such as recurrent nerve palsy and hypoparathyroidism and the supposed autonomous euthyroidism requiring no medical intervention. Other authors advocate total thyroidectomy because the incidence of iatrogenic injuries is similar to that of the subtotal procedure, and there is no risk of recurrence. Recurrence that requires further resection is a relevant factor in the choice of operation. It is difficult to evaluate the results of thyroid surgery for benign disease mainly because of the long follow-up required for complete assessment of the outcome when there may be a delay of 20 or 30 years before recurrence. According to our review of the literature, recurrence develops in as many as $14.5 \%$ of cases after subtotal resection, despite drug prophylaxis; without suppressive therapy, the rate of recurrence increases to $43 \%$. Ambrosi et al. found that recurrence was inversely related to the extent of resection. Piraneo et al. reported a $39 \%$ recurrence rate after enucleation, $27 \%$ after lobectomy, $20 \%$ after lobectomy and contralateral enucleating excision, and $4 \%$ after subtotal resection. Recurrence rates after subtotal thyroidectomy vary as much as $14 \%$. Recurrence after subtotal thyroidectomy compels patients to undergo secondary thyroidectomy.

When compared with primary thyroid surgery, secondary thyroidectomy has an increased risk of complications, such as recurrent laryngeal nerve injury and hypoparathyroidism. The best means of diminishing the complication rate is prevention through excising all pathologically altered tissue. Simple excision of a nodule or subtotal unilateral lobectomy should no longer be recommended in primary thyroid surgery. For diffuse multinodular goiter, the thyroidectomy must be sizable because the rate of recurrence is greater than $10 \%$ after 10 years and is directly related to the size of the thyroid remnant. The risk of damaging the recurrent laryngeal nerve is far higher during a 
second intervention because of the anatomic disturbance with scar tissue left behind after the first operation and degenerative changes. High rates of temporary $(15.5 \%-23.6 \%)$ and permanent $(2.6 \%-15.5 \%)$ damage of the recurrent laryngeal nerve have been reported in secondary thyroidectomy.

The indications for total thyroidectomy for managing benign thyroid disorders are not well defined; in fact, they are evolving. If a patient had an indication for total thyroidectomy that was described in the literature (ie, history of head and neck irradiation, a multinodular thyroid gland that grossly involves both lobes, locally advanced disease with compressive symptoms, and nodules highly suspected of being malignant), we performed total thyroidectomy. In our surgical department, we have a fairly low rate of indications for total thyroidectomy. In most patients, we performed near-total thyroidectomy without suspecting malignancy; however, we found papillary carcinoma in paraffin blocks for 5 patients and performed ablation. An advantage of near-total thyroidectomy over subtotal thyroidectomy is that the thyroid remnant of about $2 \mathrm{gm}$ renders it accessible to ablation if cancer is found in the specimen and obviates reoperation for completion thyroidectomy.

Permanent or temporary hypoparathyroidism is a well-known complication of total thyroidectomy. Excision of parathyroid glands and vascular insufficiency are the main causes of this complication. Although we would have liked to compare complication ratios between total thyroidectomy and near-total thyroidectomy in our surgical department, it was not appropriate because we had an insufficient number of patients who underwent total thyroidectomy. To compare our complication ratios, especially temporary hypoparathyroidism after near-total thyroidectomy with that after total thyroidectomy, we used reports of total thyroidectomy of both current and past authors. According to our review of the literature, temporary hypocalcemia ranged from $24 \%$ to $35 \%$ after total thyroidectomy. Even the lowest rate of temporary hypocalcemia after total thyroidectomy in the literature is nearly 3 times higher than the rate of temporary hypocalcemia in our series. The incidence of permanent hypoparathyroidism ranged from $0 \%$ to $0.2 \%$ after subtotal resection and from $0 \%$ to $3.8 \%$ after total resection. The incidence of permanent recurrent laryngeal nerve injuries after subtotal and total procedures varied from $0 \%$ to $1 \%$ and from $0 \%$ to $1.3 \%$, respectively. Neither permanent recurrent laryngeal nerve palsy nor permanent hypoparathyroidism occurred in our series.

We performed near-total thyroidectomy in all of our patients. The central premise of our study is the balance of leaving a small quantity of thyroid tissue adjacent to the parathyroid glands and their blood supply to minimize the risk of complications - principally, permanent and temporary hypoparathyroidism. Identification of the parathyroid glands and meticulous surgical technique to preserve parathyroid circulation are essential during near-total thyroidectomy.

The lower rate of temporary hypoparathyroidism in near-total thyroidectomy when compared with that in total thyroidectomy is an advantage because the patients need shorter postoperative hospitalization and medical treatment. This decreased cost is particularly important in our endemic region, because we have a great number of patients and limited medical services. Near-total thyroidectomy adds the advantages of total thyroidectomy (no recurrence) to those of subtotal thyroidectomy (low incidence of temporary and permanent hypoparathyroidism). In conclusion, being aware of the advantages and disadvantages of both total and subtotal thyroidectomy, we propose near-total thyroidectomy for the surgical management of multinodular goiter in endemic regions.

\section{Conclusion}

In conclusion, we recommend near-total thyroidectomy in multinodular goiter instead of total or subtotal thyroidectomy. While near-total thyroidectomy and total thyroidectomy obviate the need for completion thyroidectomy in incidentally found thyroid cancer, and while there is no difference in the rate of recurrent laryngeal nerve palsy between the two methods, neartotal thyroidectomy causes a significantly lower rate of hypoparathyroidism compared to total thyroidectomy.

\section{References}

1. Erdogan G, Erdogan MF, Emral R et al. Iodine status and goiter prevalence in Turkey before mandatory iodization. J Endocrinol Invest, 2002, 25224-228.

2. Mishra A, Agarwal G, Mishra SK. Total thyroidectomy for benign thyroid disorders in an endemic region. World $\mathrm{J}$ Surg, 2001, 25307-310.

3. Delbridge L, Guinca AI, Reeve TS Total thyroidectomy for bilateral benign multinodular goiter. Arch Surg, 1999, 1341389-1393.

4. Müller PE, Kabus S, Robens E, Spelsberg F. Indications, risks, and acceptance of total thyroidectomy for multinodular benign goiter. Surg. Today, 2001, 31958- 962.

5. Hisham AN, Azlina AF, Aina EN, Sarojah A. Total thyroidectomy: the procedure of choice for multinodular goitre. Eur. J Surg, 2001, 167403-405.

6. Pappalardo G, Guadalaxara A, Frattaroli F, MIllomei G, Falaschi P. Total compared with subtotal thyroidectomy in benign nodular disease: personal series and review of published reports. Eur J Surg. 1998, 164501-506.

7. Foster Jr, Morbidity RS. And mortality after thyroidectomy. Surg. Gynecol Obstet, 1978, 146423-429.

8. Beahrs $\mathrm{OH}$, Vandertoll DJ. Complications of secondary thyroidectomy. Surg Gynecol Obstet, 1963, 117535-539.

9. Pelizzo MR, Bernante P, Toniato A, Fassina A. Frequency of thyroid carcinoma in a recent series of 539 consecutive thyroidectomies for multinodular goiter. Tumori, 1997, 83653-655.

10. Jacobs JAl, Ballinger JJ. Total thyroidectomy: a review of 213 patients. Ann Surg. 1983, 197542-549. 